\title{
The Segmental Approximation in Multijunction Solar Cells
}

\author{
M. A. Mintairov, N. A. Kalyuzhnyy, V. V. Evstropov, V. M. Lantratov, S. A. Mintairov, M. Z. Shvarts, \\ V. M. Andreev, and A. Luque
}

\begin{abstract}
The segmental approach has been considered to analyze dark and light $I-V$ curves. The photovoltaic (PV) dependence of the open-circuit voltage $\left(V_{\mathrm{oc}}\right)$, the maximum power point voltage $\left(V_{m}\right)$, the efficiency $(\eta)$ on the photogenerated current $\left(J_{g}\right)$, or on the sunlight concentration ratio $(X)$, are analyzed, as well as other photovoltaic characteristics of multijunction solar cells. The characteristics being analyzed are split into monoexponential (linear in the semilogarithmic scale) portions, each of which is characterized by a definite value of the ideality factor $A$ and preexponential current $J_{0}$. The monoexponentiality ensures advantages, since at many steps of the analysis, one can use the analytical dependences instead of numerical methods. In this work, an experimental procedure for obtaining the necessary parameters has been proposed, and an analysis of GaInP/GaInAs/Ge triple-junction solar cell characteristics has been carried out. It has been shown that up to the sunlight concentration ratios, at which the efficiency maximum is achieved, the results of calculation of dark and light $I-V$ curves by the segmental method fit well with the experimental data. An important consequence of this work is the feasibility of acquiring the resistanceless dark and light $I-V$ curves, which can be used for obtaining the $I-V$ curves characterizing the losses in the transport part of a solar cell.
\end{abstract}

Index Terms-Concentrator photovoltaics (PV), lumped series resistance, $I-V$ curves, multijunction solar cells, photogenerated current imbalance.

\section{INTRODUCTION}

A CHIEVING higher efficiencies with multijunction (MJ) solar cells (SCs) is a topic of high priority in photovoltaic (PV) research. For this, accurate modeling is necessary [1].

To this end, numerical simulation based on the discretization and integration of the coupled differential equations that describe semiconductor devices has been applied to MJ SCs [2]-[4], as well as to distributed analytical models consisting of thousands of basic circuit units [5]-[11]. These units may be single-junction subcells, of the several semiconductors involved, tunnel junctions connecting subcells, and resistive elements joining all of them.

Nowadays, high-efficient four- and five-junction SCs are being developed, but triple-junction cells based on the latticematched $\mathrm{Ga}_{0.52} \mathrm{In}_{0.48} \mathrm{P} / \mathrm{Ga}_{0.99} \mathrm{In}_{0.01} \mathrm{As} / \mathrm{Ge}$ structure grown by the metal-organic vapor phase epitaxy technique are currently the main part of the mass production of high-efficiency MJ SCs. These cells are widely used both in space and terrestrial concentrator PV systems due to their high efficiency and high radiation resistance [12]-[14]. Although the material combination in terms of a bandgap does not give the maximum theoretical efficiency for triple-junction cells, the fact that they are lattice matched allows producing such monolithic heterostructure SCs with perfect crystalline quality and is expected to have higher long-term stability.

In this study, we apply to the MJ SCs the approach which we called segmental one. This approach is based on splitting the $V_{\mathrm{oc}}\left(J_{\mathrm{sc}}\right)$ characteristic into monoexponential segments and using the parameters of those segments to construct other relative characteristics. The segmental approach is the result of systematization of the research presented in [15]-[17]. In [15], the $I-V$ curves of single-junction GaAs and GaSb SCs and of tandem GaAs/GaSb SCs were presented as a current sum of monoexponential components. It is worth emphasizing that the ideality factors of these components were integer numbers (thus, in a single-junction SC $A=1$ for diffusion and $A=2$ for recombination mechanisms of current flow). In [16], the same concept (summing of monoexponential components having an integer ideality factors) was applied to dark $I-V$ curves of a dual-junction InGaP/GaAs SC. In this study, a different concept is presented. The main difference in this concept is that the region of $I-V$ curve, which is located between regions with integer ideality factor $A$, is presented by a segment with a fractional ideality factor. Such a segmentation is the same for the whole set of $I-V$ characteristics and characteristics that can be obtained from them, such as operating voltage on operating current $V_{m}\left(J_{m}\right)$, open-circuit voltage on short-circuit current $V_{\mathrm{oc}}\left(J_{\mathrm{sc}}\right)$, and others. It is worth noting that a similar approach has been applied for local regions near to maximum power points (MPPs) of $I-V$ curves in [17]. It was used only for determining the series resistance $R_{s}$. In the present work, this method is used in the segmental mode.

Application of the segmental approximation to MJ SCs renders the problem more involved, but at the same time increases greatly our insight on the basic cell operation permitting, as 
we believe, a deeper understanding than the more accurate numerical simulations or distributed analysis. One important advantage of the method is the way the model parameters are obtained from measurements of PV curves. In this context, PV curves are plots (usually semilogarithmic) of the current density versus voltage. One of these plots is the basic $I-V$ curve of an $\mathrm{SC}$, but others commonly used are $J_{g}\left(V_{\text {oc }}\right)$ or the dark $I-V$ curve of an SC. Still others will be described later.

Although the numeric or distributed models may be more accurate, their use is excluded from module or arrays design involving many cells (perhaps thousands). For this aim, the simplified PV curves and formulas form an approach like the one in this paper are the indispensable.

The aims of the paper are the following.

1) We propose a segmental approach to analyze PV characteristics such as $V_{\mathrm{oc}}\left(J_{q}\right), V_{m}\left(J_{q}\right), \eta(X)$, light and dark $V(J)$ curves, and others.

2) We develop a method for constructing resistanceless and complete light and dark $V(J)$ curves based on the experimental $V_{\mathrm{oc}}\left(J_{\mathrm{Sc}}\right)$ curve; practically, the photogenerated current is equal to the short-circuit current $J_{g}=J_{\mathrm{sc}}$.

3) We estimate error of segmental approximation on the example by constructing complete light $I-V$ curves of $\mathrm{Ga}_{0.52} \mathrm{In}_{0.48} \mathrm{P} / \mathrm{Ga}_{0.99} \mathrm{In}_{0.01} \mathrm{As} / \mathrm{Ge} \mathrm{SC}$ and comparing them with experimental ones.

In this study, the theoretical concepts and suitable approximations associated with the segmental model are studied in Sections II-A and B for the resistanceless case; in Appendix A, the accounting for luminescence coupling is done; in Section II$\mathrm{C}$, the lumped series resistance is added to the segmental model; and in Section II-D, the method for constructing $I-V$ curves is presented. In Section III, the segmental model is applied to the $\mathrm{Ga}_{0.52} \mathrm{In}_{0.48} \mathrm{P} / \mathrm{Ga}_{0.99} \mathrm{In}_{0.01} \mathrm{As} / \mathrm{Ge} \mathrm{SC}$. The parameters necessary for constructing resistanceless $I-V$ curves and the value of lumped linear resistance are obtained. It should be noted that the obtained resistanceless light $I-V$ curve is important for characterizing MJ SCs. A set of light $I-V$ curves has been constructed using the obtained parameters. Approximation of the transport part (characterizing losses) of MJ SC by a lumped fixed series resistance has given a good agreement with the experimental $I-V$ curves up to the sunlight concentration ratios, at which the efficiency maximum is achieved. Finally, some conclusions are drawn in Section IV.

\section{SEgmental ModEL}

\section{A. Analytical Relations for Resistanceless Case}

As followed from superposition $J=J_{g}-J_{\mathrm{dark}}[18]\left(J_{\mathrm{dark}} \equiv\right.$ $J_{\mathrm{pn}}$ and usually $J_{g}=J_{\mathrm{sc}}$ ). The PV conversion efficiency is

$$
\eta=P_{m} / P_{\text {inc }}=V_{m} \cdot J_{m} / P_{\text {inc }}
$$

where $P_{\mathrm{inc}}$ is the incident power, and $V_{m}$ and $J_{m}$ are the voltage and the current density, respectively, at MPP conditions. We shall also call these conditions "operating conditions" (we may use quotes for specific term definitions the first time the terms appear).
Instead of the variables $V_{m}$ and $J_{m}$, it may be convenient to use $J_{g}$ and the "efficiency" voltage $V_{\eta}$, the value of which is proportional to the efficiency

$$
\eta=V_{\eta} . J_{g} / P_{\mathrm{inc}}=V_{\eta} / V_{\mathrm{conv}}
$$

where

$$
V_{\text {conv }}=P_{\text {inc }} / J_{g}=\operatorname{const}\left(J_{g}\right) .
$$

Because the photogenerated current is proportional to the incident power $P_{\text {inc }}$, we can also write

$$
V_{\eta} \cdot J_{g}=V_{m} \cdot J_{m} .
$$

For the monoexponential model, the "basic" PV (superposition) curve for the resistanceless case is approximately

$$
J=J_{g}-J_{0} \exp (V / E)
$$

or

$$
V=E \ln \left(\left(J_{g}-J\right) / J_{0}\right)=\frac{k T}{q} \ln \left(\frac{J_{g}-J}{J_{0}}\right)^{A}
$$

where $E=A k T / q$ is the generalized thermal voltage, $A$ is the ideality factor, and $J_{0}$ is the preexponential (saturation) current; $q$ is the electron charge; $T$ is the absolute temperature; and $k$ is the Boltzmann constant. Equations (5a) and (5b) are valid at $V>>$.

The basic PV curve implies:

$$
J=0, V=V_{\mathrm{oc}}, \quad J_{g}=J_{0} \exp \left(V_{\mathrm{oc}} / E\right)
$$

or

$$
\begin{aligned}
& V_{\mathrm{oc}}=E \ln \left(J_{g} / J_{0}\right) \\
& J=J_{m}, V=V_{m} ; \quad J_{m}=J_{g}-J_{0} \exp \left(V_{m} / E\right)
\end{aligned}
$$

or

$$
V_{m}=E \ln \left(\frac{J_{g}-J_{m}}{J_{0}}\right) .
$$

The resistanceless dark "PV" curve is characterized by similar equations

$$
J_{g}=0, V=V_{\text {dark }}, J=-J_{\text {dark }} ; \quad J_{\text {dark }}=J_{0} \exp \left(V_{\text {dark }} / E\right)
$$

or

$$
V_{\text {dark }}=E \ln \left(J_{\text {dark }} / J_{0}\right) .
$$

Therefore, for the resistanceless case, $V_{\mathrm{oc}}\left(J_{g}\right), V_{m}\left(J_{g}-\right.$ $\left.-J_{m}\right)$, and $V_{\text {dark }}\left(J_{\mathrm{dark}}\right)$, i.e., (6b), (7b), and (8b), are coincident.

As was shown in [15] and [19], it is possible to obtain the "operating" (or MPP) PV relations:

$$
\begin{aligned}
V_{\mathrm{oc}} & =V_{m}+E \ln \left(1+V_{m} / E\right) \\
J_{g} & =J_{0}\left(1+V_{m} / E\right) \exp \left(V_{m} / E\right)
\end{aligned}
$$

or

$$
V_{m}=E \ln \left(J_{g} / J_{o}\right)-E \ln \left(1+V_{m} / E\right)
$$




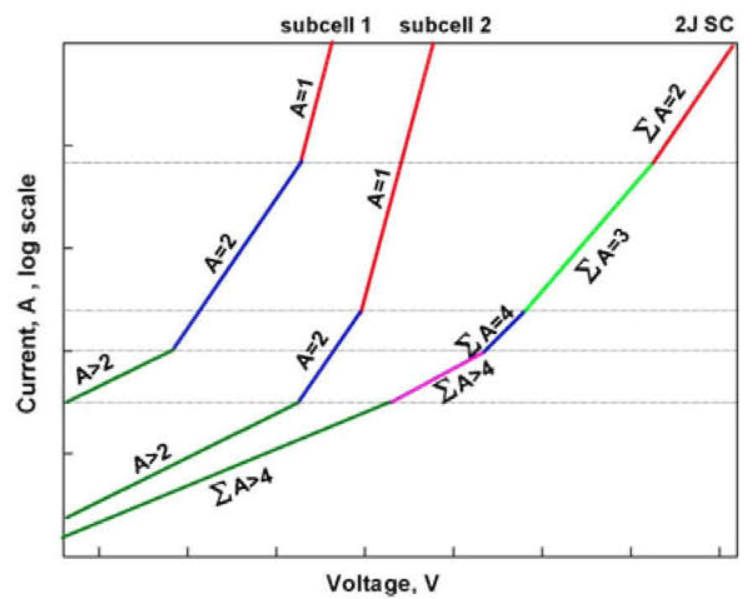

Fig. 1. Segmental approximation of single-junction SC dark $I-V$ curves and the principle of formation of a segmental dark $I-V$ curve of an MJ SC on example of $2 \mathrm{~J} \mathrm{SC}$. The horizontal lines show the current boundaries between segments with integer values of the ideality factors $A$.

and combining (4), (6a), (7a), and (9), we obtain (after a series expansion to the first degree)

$$
V_{m} \cong V_{\eta}+E .
$$

With it, we may form the "efficiency" PV curves

$$
J_{g}=J_{0}\left(2+V_{\eta} / E\right) \exp (1) \exp \left(V_{\eta} / E\right)
$$

or

$$
V_{\eta} \cong E \ln \left(J_{g} / J_{o}\right)-E \ln \left(2+V_{\eta} / E\right)-E .
$$

The comparison of the operation $V_{m}\left(J_{g}\right)$ characteristic [see (10b)] or the efficiency $V_{\eta}\left(J_{g}\right)$ characteristic [see (12b)] with dark $V_{\text {dark }}\left(J_{\text {dark }}\right)$ characteristic [see $(8 \mathrm{~b})$ ] shows that there is a practically constant voltage shift between them for the monoexponential range. As a result, these three curves are approximately parallel (in a semilogarithmic scale) for the current range, where the effect of the series resistance is negligible. The first two are separated by generalized thermal voltage $E$ [see (11)].

\section{B. Segmental Approximation}

In the segmental approximation, the dark "PV" characteristic of a single-junction SC can be represented by three segments, represented in Fig. 1, which correspond to three current components (diffusion $A=1$, recombination $A=2$, and trap tunneling $A>2$ ), which are dominant in a certain range of currents. For each segment, the $J_{\text {dark }}\left(V_{\text {dark }}\right)$ characteristic is a monoexponential characteristic. Indeed, this is an idealization. Since the subcells in the MJ SC are connected in series, the segmental approximation can be applied to the voltage sum of the subcell characteristics (see Fig. 1).

It is worth noting that the boundaries between segments with integer values of ideality factors are, practically, not sharp in contrast with Fig. 1. Therefore, in practical usage (see Appendix B, Section III, and Table I), the fractional values of the ideality factor appear. Thus, the analytical expressions and segmental approximation remain valid.
TABLE I

SEGMENT PARAMETERS: $J_{0}$-PREEXPoNENTIAL CURRENT, $E$ - GENERALIZED THermal Voltage, $A$-IDEAlity Factor

\begin{tabular}{lccc}
\hline \hline Segment & $J_{0}, \mathrm{~A} / \mathrm{cm}^{2}$ & $E, \mathrm{~V}$ & $A$ \\
\hline 1 & $4.26 \times 10^{-13}$ & 0.100 & 4.0 \\
2 & $2.74 \times 10^{-14}$ & 0.090 & 3.6 \\
3 & $1.50 \times 10^{-15}$ & 0.083 & 3.3 \\
4 & $3.18 \times 10^{-17}$ & 0.075 & 3.0 \\
\hline \hline
\end{tabular}

Consequently, the basic PV characteristic $V\left(J_{g}-J\right)$ and anyone of the PV characteristic [operating (10b), efficiency (12b)] of an MJ SC can be approximated with a set of segments [16]. In each segment, the voltage of MJ SC is formed by the sum of the voltages of the subcell characteristics. These voltages depend on the dominant current mechanism (diffusion, recombination, or tunneling) in the considered segment. As a result, the segment of basic PV characteristic has the form similar as (5b)

$$
V=\frac{k T}{q} \sum_{i=1}^{n} \ln \left[\frac{J_{g i}-J}{J_{0 i}}\right]^{A_{i}}=\frac{k T}{q} \ln \prod_{i=1}^{n}\left[\frac{J_{g i}-J}{J_{0 i}}\right]^{A_{i}}
$$

where $i$ is the subcell index, $J_{0 i}$ are the preexponential (saturation) currents, $A_{i}$ are the dominant ideality factors for the range of currents under consideration, $J_{g i}$ are the photogenerated currents, and $n$ is the number of subcells.

If the lowest photogenerated current is denoted by $J_{g}$, i.e., $J_{g}=\min \left\{J_{g 1}, J_{g 2}, \ldots, J_{g n}\right\},(13)$ can be represented as a sum of two terms [17]:

$$
V=E \ln \left[\frac{J_{g}-J}{J_{0}}\right]+V_{a} .
$$

In the first term, the resulting parameters are the following: the generalized thermal voltage is $E=A k T / q$, the ideality factor is $A=\sum_{i=1}^{n} A_{i}$, and the preexponential current is $J_{0}=$ $\sqrt[A]{\prod_{i=1}^{n} J_{o i}^{A i}}$. The second term accounts for the imbalance of the photogenerated currents:

$$
V_{a}=\frac{k T}{q} \ln \left(\prod_{i=1}^{n}\left[\frac{\kappa_{i} J_{g}-J}{J_{g}-J}\right]^{A_{i}}\right)
$$

where $\kappa_{i}=J_{g i} / J_{g} \geq 1$ are the photogenerated current imbalance coefficients, which can be obtained from spectral measurements. To calculate these parameters more accurately, the light coupling efficiency should be taken into account, but, as it is shown in Appendix A, the effect of light coupling can be neglected in practical cases (AM15D, AM0, and others).

At a full current balance (all $\kappa_{i}=1$ ), the voltage $V_{a}$ becomes zero, and the basic PV characteristic (14) takes the same form (5b) as that for a single-junction SC [17]. When the current imbalance increases ( $\kappa$ rises), $V_{a}$ becomes higher. Thus, the first term in (14) is the balanced (single-junction-like) form, and the second term $V_{a}$ characterizes the imbalance of the photogenerated currents.

In summary, a segment of the MJ SC basic PV characteristic can be described by the monoexponential model with an additional imbalance term $V_{a}$ [see (14)]. In this model, the analytical 
approaches validity for a single-junction SC can be applied to the MJ SC, but for accuracy, the $V_{a}$ voltage should be estimated.

The parameters of the segments $\left(E, J_{0}\right)$ can be obtained experimentally from the $V_{\text {oc }}\left(J_{g}\right)$ curve. The imbalance coefficients $\kappa_{i}$ can be obtained using spectral measurements. As it is shown in Appendix A, the effect of the luminescent coupling on the $I-V$ shape can be neglected (for example, correction for the open-circuit voltage is $0.2 \%$ ).

\section{Photovoltaic Determination of the Lumped Fixed Series Resistance}

One of the important factors affecting the PV conversion efficiency $\eta$ of concentrator MJ SCs of various types is the internal losses of transport covered by the term series resistance. There are different approaches [19], [20]-[22] for series resistance analysis. Here, we use a concept, for which, in practically important cases (up to the maximum efficiency), the series resistance can be represented by a fixed (independent of the illumination intensity and current) value $R_{s}$.

This concept can be easily implemented in the segmental model and allows conservation of its analytical (nonnumerical) advantages.

The series resistance in concentrator $\mathrm{SCs}$ results in the appearance of maxima of the operating voltage $V_{m}\left(J_{g}\right)$ and efficiency $\eta(X)$. Such a behavior can be used for the determination of the value of the series resistance. Then, the segment of the $V(J)$ basic PV characteristic of (14) has the form:

$$
V=E \ln \left[\frac{J_{g}-J}{J_{0}}\right]+V_{a}-J R_{s} .
$$

1) Balance Case: It is assumed that, when the effect of the series resistance on the operating point $\left(V_{m}, J_{m}\right)$ is taken into account in the balanced case $\left[V_{a}=0\right.$ in (16)], the following condition is satisfied:

$$
J_{m} \approx J_{m 0}
$$

(subscript "0" designates here the resistanceless case). Then, for the operating point, (16) takes the form

$$
V_{m}=V_{m 0}-J_{m} R_{s}=E \ln \left[\frac{J_{g}-J_{m 0}}{J_{0}}\right]-J_{m 0} R_{s} .
$$

When, in a plot $V\left(J_{g}-J_{m 0}\right)$, the first term in (18) increases, due to the increase of $\left(J_{g}-J_{m 0}\right)$, the second one decreases (since, when $J_{g}$ rises $J_{m 0}$ also rises) with a resistance-related maximum formed. In the maximum of $V_{m}$, its derivative with respect to any of three following arguments $\left(J_{g}-J_{m 0}\right), J_{g}, J_{m}$ is equal to 0 . However, it is advisable to take the derivative with respect to the argument $\left(J_{g}-J_{m 0}\right)$. It has been shown in [17] that the differentiation of (18) with consideration of (17) gives

$$
\begin{aligned}
\frac{d V_{m}}{d\left(J_{g}-J_{m}\right)} & =\frac{d V_{m}}{d J_{m}} \cdot \frac{d J_{m}}{d\left(J_{g}-J_{m}\right)} \\
& =\left(\frac{E}{J_{g}}-R_{s}\right) \cdot\left(1+\frac{V_{m 0}}{E}\right)
\end{aligned}
$$

From (19), because $d V_{m} / d\left(J_{g}-J_{m}\right)=0$, the basic relation underlying the PV method for determination of the

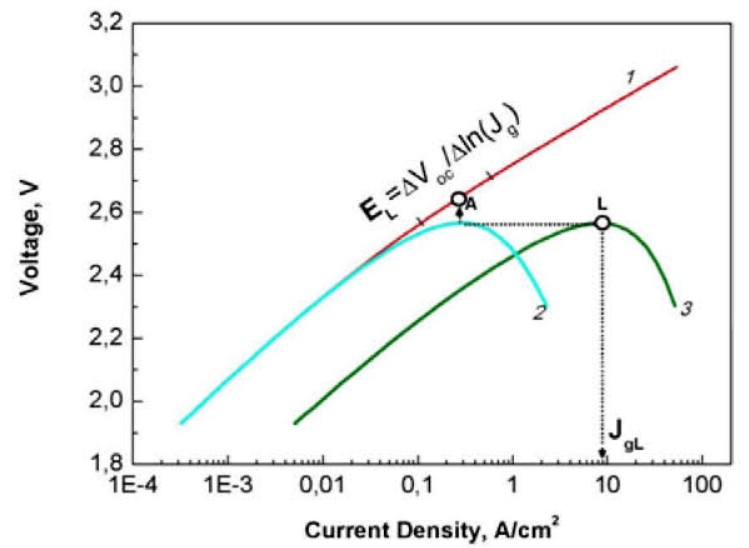

Fig. 2. Mutual positions of MJ SC PV characteristics at photogenerated current balance: (1) $V_{\mathrm{oc}}\left(J_{g}\right)$, (2) $V_{m}\left(J_{g}-J_{m}\right)$, and (3) $V_{m}\left(J_{g}\right)$. The $V_{\mathrm{oc}}\left(J_{g}\right)$ and $V_{m}\left(J_{g}-J_{m}\right)$ characteristics coincide in the resistanceless portion. The procedure of obtaining the $E_{L}$ and $J_{g L}$ needed for $R_{s}$ determination is shown. Here, the $E_{L}=0.09 \mathrm{~V}$ and $J_{g L}=9 \mathrm{~A} / \mathrm{cm}^{2}$, and therefore, due to (20), $R_{s}=$ $0.01 \Omega \cdot \mathrm{cm}^{2}$.

series resistance is

$$
R_{s}=\frac{E_{L}}{J_{g L}}
$$

where $J_{g L}$ is the photogenerated current, at which the maximum value of the operating voltage $V_{m}$ is reached, and $E_{L}$ is the value of generalized thermal voltage in (18) at the maximum of $V_{m}$. This generalized thermal voltage $E_{L}$ can be obtained from the fact that two PV characteristics (see lines 1 and 2 on Fig. 2), $V_{\text {oc }}\left(J_{g}\right)$ [see (6b)] and $V_{m}\left(J_{g}-J_{m}\right)$ [see (18)] have the same functional dependence, but (6b) has a resistanceless form, and (18) has a resistive form. Thus, they are split into the same segments. Therefore, $E_{L}$ is the generalized thermal voltage $E$ of the segment, at which the $V_{m}$ maximum is situated. Experimentally, the required value of $E_{L}$ is equal to the logarithmic slope (ratio $\Delta V_{\text {oc }} / \Delta \ln \left(J_{g}\right)$ ) for this segment (see Fig. 2, point $A$ ).

Fig. 2 illustrates the method for finding the series resistance from the expression of (20) with using the $V_{\text {oc }}\left(J_{g}\right), V_{m}\left(J_{g}-\right.$ $\left.J_{m}\right)$ and $V_{m}\left(J_{g}\right)$ curves described above. The quantity $J_{g L}$ is the current at the point $L$, and $E_{L}$ is found from the logarithmic slope of the characteristics $V_{\text {oc }}\left(J_{g}\right)$ of the segment, which have the point $A$.

2) Imbalance Case: For the imbalance case, in contrast with the balance one, it is necessary to take into account the additional imbalance voltage $V_{a}(J)$ of (14) and (15). Because $V_{m}\left(J_{g}\right)$ and $V_{\text {oc }}\left(J_{g}\right)$ are used to find $R_{s}$ with (20), it is necessary to determine the corresponding imbalance voltage corrections $\left(V_{a, m}, V_{a, o c}\right)$.

It follows from (15) that in the open-circuit mode $(J=$ $\left.0, V_{a}=V_{a, o c}\right)$, the imbalance correction of the open-circuit voltage has the form

$$
V_{a, o c}=\frac{k T}{q} \ln \left(\prod_{i=1}^{n}\left[\kappa_{i}\right]^{A_{i}}\right)
$$

i.e., it is strictly constant.

In the operating mode $\left(J=J_{m}, V_{a}=V_{a, m}\right)$, as is shown in [17], the imbalance correction of the operating voltage $V_{a, m}$ can 
be approximated by a constant in a limited range of photogenerated currents.

The fact that $V_{a, o c}$ is a constant and $V_{a, m}$ is approximated by a constant in a limited range of currents leads to the derivation of the basic formula of (20), which remains unchanged. Accordingly, the procedure for finding the local generalized thermal voltage $E_{L}$ from the logarithmic slope of the $V_{\text {oc }}\left(J_{g}\right)$ characteristic at the point $A$ and the photogenerated current $J_{g L}$ at the point $L$ is unchanged in spite of that curves 1 and 2 (see Fig. 2) do not coincide in the imbalance case. This was verified experimentally in [17].

This method allows determining the fixed lumped series resistance of the transport part, which can be used in the segmental model for the description of the MJ SC $I-V$ curves. Such a resistance, as well as the other parameters of the segmental model, can be obtained experimentally.

\section{Construction of the I-V Curves}

The segmental approach allows constructing the $I-V$ curves starting from the experimental $V_{\mathrm{oc}}\left(J_{\mathrm{sc}}\right)$ curve. The constructing process consists of two stages: acquisition of the resistanceless $I-V$ curve and the resistive voltage correction, which is approximated by a fixed lumped series resistance (see Section II-C).

Below, the following sequence of actions in constructing the resistanceless $I-V$ curve is outlined.

1) We split the experimental $V_{\mathrm{oc}}\left(J_{\mathrm{sc}}\right)$ (usually $J_{\mathrm{sc}}=J_{g}$ ) characteristic (in the logarithmic scale by current and linear scale by voltage) into straight-line segments (piecewise linear approximation). Splitting is performed in such a way that deviation of points from the rectilinear segments would not exceed the predetermined value. In this study, this value was $1 \%$. It is desirable to select segments with integer values of the ideality coefficient $A$.

2) We calculate, by means of (21), for each segment of the voltage shift $V_{a, o c}$. The set of photogenerated currents of subcells is obtained from the experimental spectral external quantum efficiency, and therefore, the set of imbalance coefficients $\kappa_{i}$ is also obtained. A set of subcell ideality factors $A_{i}$ can be obtained either from a priori physical considerations, from an additional analysis of the experimental data (for example, from luminescence measurements) or by rating. Details of the rating are given in Appendix B.

3) We subtract of calculated voltage shifts $V_{a, o c}$ from the experimental $V_{\mathrm{oc}}\left(J_{\mathrm{sc}}\right)$ characteristic, and as a result, obtain the segmental balanced $V_{\text {oc }}\left(J_{\mathrm{sc}}\right)$ curve which coincides with resistanceless dark $V_{\text {dark }}\left(J_{\text {dark }}\right)$ [see (6b) and (8b)].

4) We current shift all segments of the obtained $I-V$ curve by the value $J_{\mathrm{sc}}=\min \left\{J_{g 1}, J_{g 2}, \ldots, J_{g n}\right\}$; as a result, the light $I-V$ curve is obtained, which corresponds to the conventional case of equality of all photogenerated currents to one and the same value.

5) We calculate of the voltage correction $V_{a}(J)$ for all segments by means of (15). As mentioned above (2 in this list), a set of photogenerated currents and subcell ideality

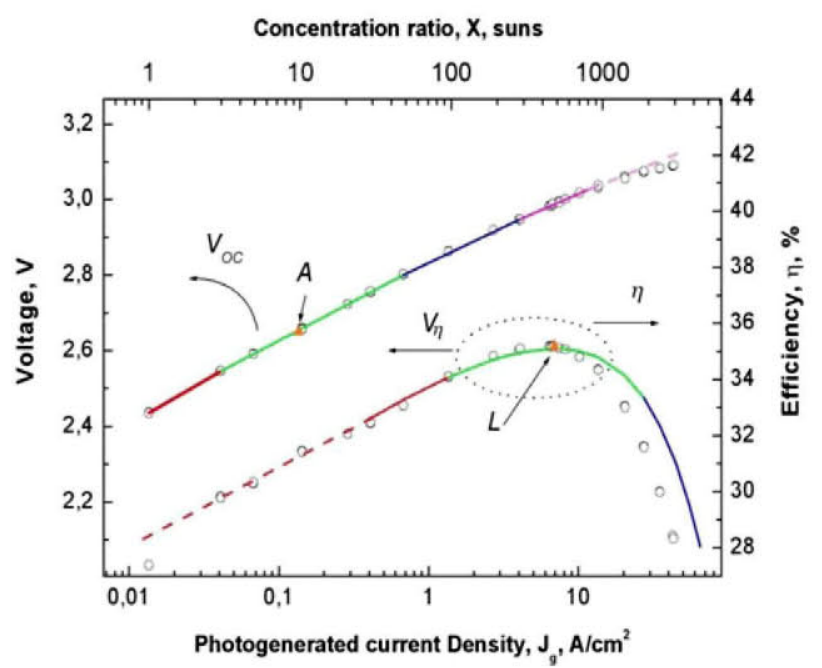

Fig. 3. Experimental dependences $V_{\circ \mathrm{oc}}(X), \eta(X)$, and $V_{\eta}(X)$ (circles). Rated dependences $\eta(X)$ by the monoexponential segmental model (multicolored line). Different colors of the segments on the rated dependence $\eta(X)$ correspond to that of the segments on the experimental dependence $V_{\mathrm{OC}}(X)$. Note that $\eta$ is proportional to $V \eta$ and $X$ to $J_{g}$. The parameters and numbering of the segments are presented in Table I (1-red, 2-green, 3-blue, 4-violet).

factors are used. As a result, the resistanceless light $I-V$ curve is obtained.

The complete resulting $I-V$ curve is obtained by means of the voltage correction equal to the voltage on transport part of SC. In the proposed method, such a correction is done by approximating the transport part by the lumped fixed series resistance (see Section II-C). Application of the segmental approach for $\mathrm{GaInP/GaInAs/Ge} \mathrm{SC}$ and obtaining a set of $I-V$ curves by means of the described above steps for different sunlight concentration ratios is described in the following section.

\section{APPLICATION OF SEGMENTAL APPROXIMATION FOR TRIPLE-JUNCTION SOLAR CELLS}

For using the segmental approach, the parameters of segments $J_{0}$ and $E$, photogenerated current subcell imbalance coefficients $\kappa$, and fixed lumped series resistance $R_{s}$ have been determined.

The following values of the photogenerated current have been obtained from spectral measurements: $J_{g(\mathrm{GaInP})}=13.72$ $\mathrm{mA} / \mathrm{cm}^{2}, J_{g(\mathrm{GaInAs})}=13.48 \mathrm{~mA} / \mathrm{cm}^{2}$, and $J_{g(\mathrm{Ge})}=20.84$ $\mathrm{mA} / \mathrm{cm}^{2}$. According to these values, the imbalance coefficients of the GaInP/GaInAs/Ge SC are the following: $\kappa_{\mathrm{GaInP}}=1.01$, $\kappa_{\mathrm{GaInAs}}=1$, and $\kappa_{\mathrm{Ge}}=1.49$.

The segment parameters can be conveniently obtained by using the $V_{\text {oc }}\left(J_{g}\right)$ curve shifted by the value of the additional voltage $V_{a, \text { oc }}$ of (21). It should be noted that in our case, $V_{a}$ depends only on $\kappa_{\mathrm{Ge}}$, because just this imbalance coefficient differs from 1 noticeably. According to (21), $V_{a, o c}=10 \mathrm{mV}$ (the ideality factor $A_{\mathrm{Ge}}=1$ [23]). The shift of the $V_{\mathrm{oc}}\left(J_{g}\right)$ curve for this value was done, and the $J_{0}$ and $E$ parameters for segments were found (see Table I).

The segments are marked by colors in Fig. 3; two of them ( 1 and 4 ) have an integer-valued ideality factor (respectively, 4 and 3 ). 


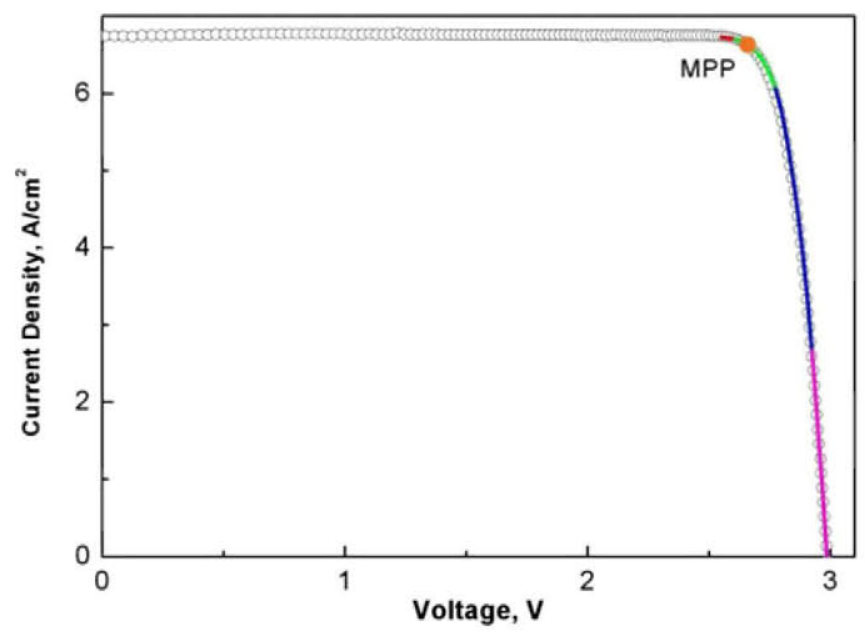

Fig. 4. Experimental (symbols) and obtained by segment method $I-V$ curves (colored lines) for the sunlight concentration ratio $(X=500)$ at which the efficiency is achieved maximum value (Fig. 3 point L). Segments have the same color as in Fig. 3. The relative error in the efficiency in the MPP (orange circle) is of the order of $0.05 \%$

The method described in Section II-C has been applied for determining the series resistance. The value $J_{g L}$ was equal to $6.92 \mathrm{~A} / \mathrm{cm}^{2}$ (see Fig. 3, point $L$ ), and the corresponding value was equal to $E_{L}=0.09 \mathrm{~V}$ (see Table I segment 2 and Fig. 3 green line). Therefore, according to (20), the lumped fixed series resistance $R_{s}=0.013 \Omega \cdot \mathrm{cm}^{2}$.

As is pointed out in Section II-D, it is necessary to preset a set of subcell ideality factor values. Such a set has been found by rating. The results are presented in Appendix B.

Thus, all data for the monoexponential segmental model were found, and they were used for the MJ SC $I-V$ curves approximation. The calculation results are presented in Figs. 3 and 4. Fig. 3 shows the results of calculation of the $V_{\mathrm{oc}}(X), \eta(X)$, and $V_{\eta}(X)$. It is seen that, at sunlight concentration of up to 500 $\left(J_{g L}=6.92 \mathrm{~A} / \mathrm{cm}^{2}\right)$, the approximation gives a good fit to the experimental data.

It should be pointed out that the rated curve $V_{\mathrm{oc}}\left(J_{\mathrm{sc}}\right)$ (see Fig. 3) does not fit to the experimental data at $X>1000$. This can be associated with heating the specimen during recording $I-$ $V$ characteristics, or with the presence in the MJ SC structure an objects generating a counter electromotive force (for example, tunnel diodes can separate charge carriers generating electromotive force). It should be also noted that the rating does not take into account tunneling mechanisms of current flow. For this reason, there is a discrepancy of rated $\eta(X)$ curve at $X \approx 1$. However, this is not very critical for describing $I-V$ characteristics of SCs working on concentration conditions (here at $X>3)$.

Fig. 4 shows the result of calculation of an $I-V$ curve by the segmental approximation method (see Section II-D). The calculation was performed for the sunlight concentration ratio of 500, at which the efficiency maximum is achieved. It is clear that coincidence of the presented characteristics is quite good. For example, the relative error in the efficiency in the MPP is of the order of $0.05 \%$. Correspondingly, the absolute error is
$0.2 \%$, which is less than the measurement error (of the order of $\pm 0.5 \%$ ).

At concentration ratios greater than 500, the approximation of the transport part by the lumped fixed series resistance is not sufficient, which is clearly seen from Fig. 3. This may be caused by the presence of a tunnel junction, a spreading resistance or heterointerfaces. Preliminary estimates show that it is most likely that the main contribution comes from the spreading resistance. However, it should be noted that the method for constructing the resistanceless $I-V$ curve remains valid.

\section{CONCLUSION}

A segmental approach has been considered, which allows analyzing PV characteristics, such as $V_{\text {oc }}\left(J_{g}\right), V_{m}\left(J_{g}\right)$, $V_{\eta}\left(J_{g}\right), \eta(X)$, light and dark $V(J)$ curves, and others. The segmental approach uses the advantages that are inherent in the monoexponential (correspondingly logarithmic) dependence being contained in the resistanceless characteristics. The main advantage consists in the possibility to use in many cases the analytical dependencies instead of numerical methods. Such a monoexponential dependence is the most effective, if the preexponential (saturation) currents (diffusion and, particularly, recombination ones) can be neglected compared with real currents through a p-n junction, $\left(J_{0}<<\left(J_{g}-J\right)=J_{\text {pn }}=J_{\text {dark }}\right)$. This condition is always fulfilled in modern solar single-crystalline cells. Besides, a possibility appears to transfer equations valid for single-junction SC to MJ SCs.

A method has been proposed to obtain resistanceless $I-V$ characteristics (with and without illumination) starting from experimental $V_{\mathrm{oc}}\left(J_{\mathrm{sc}}\right)$ characteristic (note: practically, $J_{\mathrm{sc}}=J_{g}$ ). In this method, the resistanceless $V_{\mathrm{oc}}\left(J_{g}\right)$ characteristic is split. in the beginning into exponential segments (straight line in a semilogarithmic scale). Other resistanceless characteristics are constructed from the obtained segments. These characteristics are important, since, first, they are basic ones for characterizing PV p-n junctions, for example, for determining the preexponential currents in the segments, which have integer ideality factors ( $A=1,2,3,4$, and so on). Second, they allow estimating a potential efficiency, which can be obtained from a device semiconductor structure. Third, they allow separating the $I-V$ curve of the MJ SC transport part. Such a separation is performed by the voltage subtraction of the constructed resistanceless $I-V$ curve from the experimental one. In this case, one can analyze the $I-V$ curve of the transport part and elaborate more accurate approximations of this characteristic.

In this study, a method for determining the lumped fixed series resistance is presented. This method amplifies the method for obtaining the resistanceless $I-V$ curves because together, both methods allow the description of experimental PV characteristics. They have been applied for GaInP/GaInAs/Ge SCs. A good fit has been obtained to experimental characteristics up to the concentration ratios, at which the maximum efficiency is achieved. For the $I-V$ curve obtained at such ratios, a relative efficiency error in the MPP point is of the order of $0.05 \%$. Parameters of the segments and the lumped fixed series resistance have been found. 


\section{APPENDIX A \\ ACCOUNT FOR LUMINESCENT COUPLING}

Photons, which are generated owing to electroluminescence inside the MJ SC structure, give an additional contribution to the photogenerated current imbalance. The electroluminescence photon emission of a p-n junction depends on the current flowing through it $J_{\mathrm{pn}(i)}=\left(J_{g(i)}-J\right)$. The photons generated by the p-n junction induce an additional photogenerated current in the adjacent p-n junction with a narrower band gap. As a result, a correlation arises between the current flowing through the p-n junction $J_{\mathrm{pn}(i)}=\left(J_{g(i)}-J\right)$ and the photogenerated current in next one $J_{g(i+1)}$. As is shown in [24] and [25], accounting for the luminescent coupling can be performed by simple cycling calculations. Principally, the procedure can be applied also for (15). This can be done by introducing the dependence of $\kappa_{(i+1)}$ on $\left(J_{g(i)}-J\right)$; however, calculation of $V_{a}$ will be more cumbersome. On the other hand, this effect for conventional MJ SCs does not strongly affect the $I-V$ characteristic shape. It is clear from the estimation for a typical triple-junction SC according to [24]. For this, it is necessary to preset the values of photogenerated and preexponential currents for all p-n junctions and also the coupling yield of the first (GaInP/GaInAs) and the second (GaInAs/Ge) pairs. Coupling yield can be taken from [26]: 7\% and 50\% for the first and second pairs, respectively. Values of the preexponential and photogenerated currents for subcells are taken as typical: $\left(1 \times 10^{-25}, 1 \times 10^{-20}, 1 \times 10^{-6}\right) \mathrm{A} / \mathrm{cm}^{2}$-diffusion preexponential currents; $\left(1 \times 10^{-13}, 1 \times 10^{-10}, \ll 1 \times 10^{-6}\right)$ $\mathrm{A} / \mathrm{cm}^{2}$-recombination preexponential currents; and $(14,14$, 21) $\mathrm{mA} / \mathrm{cm}^{2}-1$-sun photogenerated currents. The series resistance can be taken from [17] as equal to $13.7 \mathrm{~m} \Omega \cdot \mathrm{cm}^{2}$. Performing calculations similar to those [24] for the case of AM1.5D in two versions (with and without accounting for the luminescent coupling, i.e., when the coupling yield is equal or not equal to zero), we obtain that the efficiency maximum in both versions will be achieved at the concentration ratio of 500 . In this case, the open-circuit voltage in accounting for the luminescent coupling is $3.086 \mathrm{~V}$ and without it $-3.079 \mathrm{~V}$, voltages in the point of the maximum generated power are 2.723 and $2.722 \mathrm{~V}$, and the efficiencies are $37.02 \%$ and $37.00 \%$, respectively. It is seen from the presented values that the observed distinctions between the results are small, and hence, the effect of the luminescent coupling on the $I-V$ curve shape can be neglected.

\section{APPENDIX B}

\section{Rating a SET OF SUbCell Ideality FaCtors}

The present calculation assumes that any segment with the integer ideality factor $A$ (for example, $A=3$ and $A=4$, Table I) is a sum of integer $A_{i}$ (where $i$ is the subcell index), and the sum can be realized in several variants. For example, for a triplejunction SC, $A=3$-one variant (all $A_{i}=1$ ) -and $A=4-$ three variants (one of $A_{i}=2$ ). The amount of ways of transitions between the initial and final variants is finite (for example, transition from the segment with $A=4$ to one with $A=3$ can be performed in three ways). Calculation of the $I-V$ curves is carried out for all possible ways. The way that better corresponds to the experimental curve is chosen. Below, an example of obtaining a set of $A_{i}$ for the triple-junction $\mathrm{GaInP} / \mathrm{GaInAs} / \mathrm{Ge} \mathrm{SC}$ considered in Section III is presented.

It is clear from Table I that there are two segments with integer values of $A$ (segment 1 with $A=4$ and segment 4 with $A=3$ ). As is pointed out above, the amount of the variants for the segment with $A=3$ is equal to unity. This one corresponds $\mathrm{A}_{\mathrm{GaInP}}=1, \mathrm{~A}_{\mathrm{GaInAs}}=1, \mathrm{~A}_{\mathrm{Ge}}=1$. For the segment with $A=4$, there are two variants: One is $A_{\mathrm{GaInP}}=$ 2, $A_{\mathrm{GaInAs}}=1, A_{\mathrm{Ge}}=1$, and other is $A_{\mathrm{GaInP}}=1, A_{\mathrm{GaInAs}}=$ $2, A_{\mathrm{Ge}}=1$. Note that the third variant with $A_{\mathrm{Ge}}=2$ is absent, which is associated with that Ge subcell ideality factor is always $A_{\mathrm{Ge}}=1$ [23]. Therefore, three ways of the transition from $A=4$ to $A=3$ are formed. It has been established in carrying out the calculation that the best fit to the experimental data was for the second way, i.e., when the transition from $\mathrm{A}=4\left(\mathrm{~A}_{\mathrm{GaInP}}=1, \mathrm{~A}_{\mathrm{GaInAs}}=2, \mathrm{~A}_{\mathrm{Ge}}=1\right)$ to $A=3$ $\left(\mathrm{A}_{\mathrm{GaInP}}=1, \mathrm{~A}_{\mathrm{GaInAs}}=2, \mathrm{~A}_{\mathrm{Ge}}=1\right)$ is performed. Thus, it has been determined that the ideality factor for GaInP and Ge subcells and for all segments is equal to $\mathrm{A}_{\mathrm{Ge}}=\mathrm{A}_{\mathrm{GaInP}}=1$, and for the GaInAs subcell $\mathrm{A}_{\mathrm{GaIn} A s}$ changes from 2 (segment 1) to 1 (segment 4 ).

\section{ACKNOWLEDGMENT}

The authors would like to thank G.V. Il'menkov for useful discussions and $\mathrm{N}$. K. Timoshina for the measurements of $I-V$ curves and PV dependences.

\section{REFERENCES}

[1] I. Rey-Stolle, C. Algora, I. Garcia, M. Baudrit, P. Espinet, B. Galiana, and E. Barrigon, "Simulating III-V concentrator solar cells: A comparison of advantages and limitations of lumped analytical models; distributed ana1ytical models and numerical simulation," in Proc. 34th IEEE Photovoltaic Spec. Conf., 2009 , p. 6.

[2] S. Michael, "A novel approach for the modeling of advanced photovoltaic devices using the SILVACO/ATLAS virtual wafer fabrication tools," Sol. Energy Mater. Sol. Cells, vol. 87, pp. 771-784, May 2005.

[3] M. Baudrit, C. Algora, and IEEE, "Modeling of GaInP/GaAs dual-junction solar cells including tunnel junction," in Proc. 33rd IEEE Photovoltaic Spec. Conf., 2008, vol. 1-4, pp. 576-580.

[4] A. Hermle, G. Letay, S. P. Philipps, and A. W. Bett, "Numerical simulation of tunnel diodes for multi-junction solar cells," Prog. Photovoltaics, vol. 16, pp. 409-418, Aug. 2008.

[5] H. C. Hamaker, "Computer modeling study of the effects of inhomogeneous doping and of composition in GaAs solart-cell devices," J. Appl Phys., vol. 58, pp. 2344-2351, 1985.

[6] K. Araki and M. Yamaguchi, "Extended distributed model for analysis of non-ideal concentration operation," Sol. Energy Mater: Sol. Cells, vol. 75, pp. $467-473$, Feb. 2003.

[7] I. Garcia, C. Algora, I. Rey-Stolle, and B. Galiana, "Study of nono-uniform light profiles on high concentration III-V solar cells using quasi-3d distributed models," in Proc. 33rd IEEE Photovoltaic Spec. Conf., 2008 vol. 1-4, pp. 351-356.

[8] B. Galiana, C. Algora, I. Rey-Stolle, and I. G. Vara, "A 3-D model for concentrator solar cells based on distributed circuit units," IEEE Trans. Electron Devices, vol. 52, no. 12, pp. 2552-2558, Dec 2005.

[9] I. Garcia, P. Espinet-Gonzalez, I. Rey-Stolle, and C. Algora, "Analysis of chromatic aberration effects in triple-junction solar cells using advanced distributed models," IEEE J. Photovoltaics, vol. 1, no. 2, pp. 219-224, Oct. 2011.

[10] N. A. Kalyuzhnyy, A. S. Gudovskikh, V. V. Evstropov, V. M. Lantratov, S. A. Mintairov, N. K. Timoshina, M. Z. Shvarts, and V. M. Andreev, "Current flow and efficiency of Ge P-N junctions in triple-junction GaInP/Ga(In)As/Ge solar cells for space applications," in Proc. 25th Eur. 
Photovoltaic Sol. Energy Conf. Exhib./5th World Conf. Photovoltaic Energy Convers., 2010, pp. 865-871.

[11] V. M. Emelyanov, N. A. Kalyuzhnyy, M. A. Mintairov, S. A. Mintairov, M. Z. Shvarts, and V. M. Lantratov, "Distributed resistance effects simulation in concentrator MJ SCS using 3D-network model," in Proc. 25th Eur. Photovoltaic Sol. Energy Conf. Exhib./5th World Conf. Photovoltaic Energy Convers., 2010, pp. 406-411.

[12] M. A. Green, K. Emery, Y. Hishikawa, W. Warta, and E. D. Dunlop, "Solar cell efficiency tables (version 43)," Progress Photovoltaics, vol. 22, pp. 1-9, Jan. 2014.

[13] R. R. King, D. C. Law, K. M. Edmondson, C. M. Fetzer, G. S. Kinsey, H. Yoon, R. A. Sherif, and N. H. Karam, "40\% efficient metamorphic GaInP/GaInAs/Ge multijunction solar cells," Appl. Phys. Lett., vol. 90 p. 183516, Apr. 2007.

[14] F. Dimroth, M. Grave, P. Beutel, U. Fiedeler, C. Karcher, T. N. D. Tibbits, E. Oliva, G. Siefer, M. Schachtner, A. Wekkeli, A. W. Bett, R. Krause, M. Piccin, N. Blanc, C. Drazek, E. Guiot, B. Ghyselen, T. Salvetat, A. Tauzin, T. Signamarcheix, A. Dobrich, T. Hannappel, and K. Schwarzburg, "Wafer bonded four-junction GaInP/GaAs//GaInAsP/GaInAs concentrator solar cells with $44.7 \%$ efficiency," Prog. Photovoltaics, vol. 22, pp. 277-282, Mar. 2014.

[15] V. M. Andreev, V. V. Evstropov, V. S. Kalinovsky, V. M. Lantratov, and V. P. Khvostikov, "Current flow and potential efficiency of solar cells based on GaAs and GaSb p-n junctions," Semiconductors, vol. 43, pp. 644-651, May 2009.

[16] V. S. Kalinovsky, V. V. Evstropov, N. A. Kalyuzhnyy, V. M. Lantrator, S. A. Mintairov, and V. M. Andreev, "Structure of the dark I-V characteristic of multijunction solar cells and their efficiency," in Proc. 23th Eur: Photovoltaic Sol. Energy Conf., Valencia, Spain, 2008, pp. 773-776.

[17] M. A. Mintairov, V. V. Evstropov, N. A. Kalyuzhnyi, C. A. Mintairov, N. K. Timoshina, M. Z. Shvartz, and V. M. Lantratov, "Photoelectric determination of the series resistance of multijunction solar cells," Semiconductors, vol. 46, pp. 1051-1058, Aug. 2012.

[18] N. G. Tarr and D. L. Pulfrey, "Investigation of dark current and photocurrent superposition in photo-voltaic devices" Solid-State Electron., vol. 22 pp. $265-270,1979$.
[19] V. M. Andreev, V. A. Grilikhes, and V. D. Rumyantsev, Photovoltaic Conversion of Concentrated Sunlight. New York, NY, USA: Wiley, 1997.

[20] B. Galiana, C. Algora, and I. Rey-Stolle, "Explanation for the dark IV curve of III-V concentrator solar cells," Prog. Photovoltaics, vol. 16, pp. 331-338, Jun. 2008.

[21] D. Aiken, M. Stan, G. Girard, S. Endicter, and P. Sharps, "Development and testing of III-V multijunction-based terrestrial concentrator modules," in Proc. Conf. Rec. 31st IEEE Photovoltaic Spec. Conf., 2005, pp. $743-$ 746

[22] V. M. Emelyanov, N. A. Kalyuzhnyy, M. A. Mintairov, S. A. Mintairov, M. Z. Shvarts, and V. M. Lantratov, "Distributed resistance effects simulation in concentrator MJ SCS using 3D-network model," in Proc. 25th Eur. Photovoltaic Sol. Energy Conf. Exhib./5th World Conf. Photovoltaic Energy Convers., 2010, pp. 406-411.

[23] N. A. Kalyuzhnyy, S. S. Gudovskikh, V. V. Evstropov, V. M. Lantratov, S. A. Mintairov, N. K. Timoshina, M. Z. Shvarts, and V. M. Andreev, "Germanium subcells for multijunction GaInP/GaInAs/Ge solar cells," Semiconductors, vol. 44, pp. 1520-1528, 2010.

[24] M. A. Mintairov, V. V. Evstropov, N. A. Kalyuzhnyy, S. A. Mintairov, M. Z. Shvarts, and N. K. Timoshina "Photogenerated currents imbalance in MJ SC: IV curve and efficiency variations," presented at the 10th Int. Conf. Concentrator Photovoltaics, Albuquerque, NM, USA, 2014.

[25] D. J. Friedman, J. F. Geisz, and M. A. Steiner, "Analysis of multijunction solar cell current-voltage characteristics in the presence of luminescent coupling,"IEEE J. Photovoltaics, vol. 3, no. 4, pp. 1429-1436, Oct. 2013.

[26] M. Z. Shvarts, M. A. Mintairov, V. M. Emelyanov, V. V. Evstropov, V. M. Lantratov, and N. K. Timoshina, "Method for ditect measurements of luminescent coupling efficiency in concentrator MJ SCs," in Proc. AIP Conf., Miyazaki, Japan, 2013, pp. 147-151.

Authors' photographs and biographies not available at the time of publication. 